\title{
Double gyroid structures made of asymmetric dimers
}

\author{
Joanna Maria Wolska*, Damian Pociecha, Jozef Mieczkowski, Ewa \\ Gorecka
}

Department of Chemistry, Warsaw University, Pasteura 1, 02-093 Warsaw, Poland jokos@chem.uw.edu.pl Tel: +48228220211

\section{Experimental section}

Substrates and solvents were obtained from Sigma-Aldrich. Substrates were used without purification; solvents were dried over activated $3 \AA$ molecular sieves for $24 \mathrm{~h}$. Presented reactions were carried out under an argon atmosphere with using a magnetic stirring hotplate. All products were purified by column chromatography with Rushan Taiyang silica gel 60 (230-400 mesh). Analytical thin-layer chromatography (TLC) was performed using Silica Gel $60 \AA$ F254 (Merck) pre-coated plastic/alumina plates $(0.25$ mm thickness) and visualized using UV lamp (254 nm) and iodine vapour. Presented yields refer to chromatographically and spectroscopically ( ${ }^{1} \mathrm{H}$ NMR) homogeneous materials.

The ${ }^{1} \mathrm{H}$ NMR and ${ }^{13} \mathrm{C}$ NMR spectra were recorded at NMR Varian Unity Plus $200 \mathrm{MHz}$ or at NMR Bruker AVANCE $300 \mathrm{MHz}$. Proton chemical shifts were reported in ppm ( $\delta$ ) relative to internal standard - tetramethylsilane ( TMS $\delta, 0.00 \mathrm{ppm}$ ). Carbon chemical shifts are reported in ppm $(\delta)$ relative to the residual solvent signal $\left(\mathrm{CDCl}_{3}, \delta\right.$ 77.0). Data are reported as follows: chemical shift, integration, multiplicity ( $\mathrm{s}=$ singlet, $\mathrm{d}=$ doublet, $\mathrm{dd}=$ doublet of doublets, $\mathrm{t}=$ triplet, $\mathrm{q}=$ quartet, $\mathrm{br}=$ broad, $\mathrm{m}=$ multiplet ), and coupling constants $(\mathrm{Hz})$.

Temperatures and thermal effects of the phase transitions were determined by calorimetric studies using TA Q200 calorimeter, samples of mass 1-3 mg were sealed in aluminum pans and kept in nitrogen atmosphere during measurement. Both heating and 
cooling scans with rate $5-10 \mathrm{~K} / \mathrm{min}$ were applied. The optical studies were performed using Zeiss Imager A2m polarizing microscope equipped with Linkam heating stage.

The small angle X-ray diffraction patterns were obtained with Bruker Nanostar system (parallel beam of $\mathrm{CuK} \alpha$ radiation formed by cross-coupled Goebel mirrors and 3-pinhole collimation system, area detector VANTEC 2000). The temperature of the sample was controlled with precision 0.1 degree. The signal intensities vs. wavevector $\mathrm{q}$ were obtained through integration of the $2 \mathrm{D}$ patterns over azimuthal angle. The broad angle diffraction patterns were collected with Bruker GADDS system ( $\mathrm{CuK} \alpha$ radiation, Goebel mirror, point beam collimator) equipped with area detector VANTEC 2000. XRD patterns were analysed with Bruker Topas 3 software, allowing precise determination of individual peak positions and intensities (peak phase) as well as fitting of the whole pattern in order to confirm assumed symmetry of the structure and determine crystallographic unit cell parameters ( $h k l$ phase). In the latter case the peak positions are not independent parameters, they are calculated from the unit cell size and symmetry.

\section{Synthesis and characterization of compounds $L$}

Synthesis and characterization of compounds $\mathbf{L}$, showed on Scheme 1. (Figure 1. in main text), is presented for compound $\mathbf{L} \mathbf{1}(n=5, R=H)$.

\section{Synthesis of the methyl 3,4,5-tridodecyloxybenzoate (1):}

Into the solution of $18,5 \mathrm{~g}(0,1 \mathrm{~mol})$ of methyl $3,4,5$-trihydroxybenzoate in $750 \mathrm{ml}$ of N,N-dimethylformamide a 49,6g $(0,36 \mathrm{~mol})$ of anhydrous potassium carbonate and $59,8 \mathrm{~g}(0.36 \mathrm{~mol})$ of potassium iodide was added. Next $89,6 \mathrm{~g}(0,36 \mathrm{~mol})$ of 1 bromododecane was added dropwise. The mixture was vigorously stirred in temperature of $80^{\circ} \mathrm{C}$ for $24 \mathrm{~h}$. After cooling to the room temperature the mixture was poured into the $1500 \mathrm{ml}$ of cold water with ice. The precipitate was filtered and recrystallized twice from ethanol affording 
$62,4 \mathrm{~g}(90,8 \%)$ of product was obtained as a white solid. Elemental analysis for $\mathrm{C}_{44} \mathrm{H}_{80} \mathrm{O}_{5}$ $(\mathrm{M}=688,60) \mathrm{C} 76,69 ; \mathrm{H} \mathrm{11,70 \%}$, calc., found: $\mathrm{C} 76,62 ; \mathrm{H} \mathrm{11,72 \% ;}{ }^{1} \mathrm{H} \mathrm{NMR}:\left(200 \mathrm{MHz}, \mathrm{CDCl}_{3}\right)$ $\delta: 6.69(2 \mathrm{H}, \mathrm{s}), 4.09-3.96(6 \mathrm{H}, \mathrm{m}) ; 1.92-1.66(6 \mathrm{H}, \mathrm{m}) ; 1.58-1.14(54 \mathrm{H}, \mathrm{m}) ; 0.94-0.80$ $(9 \mathrm{H}, \mathrm{m})$.
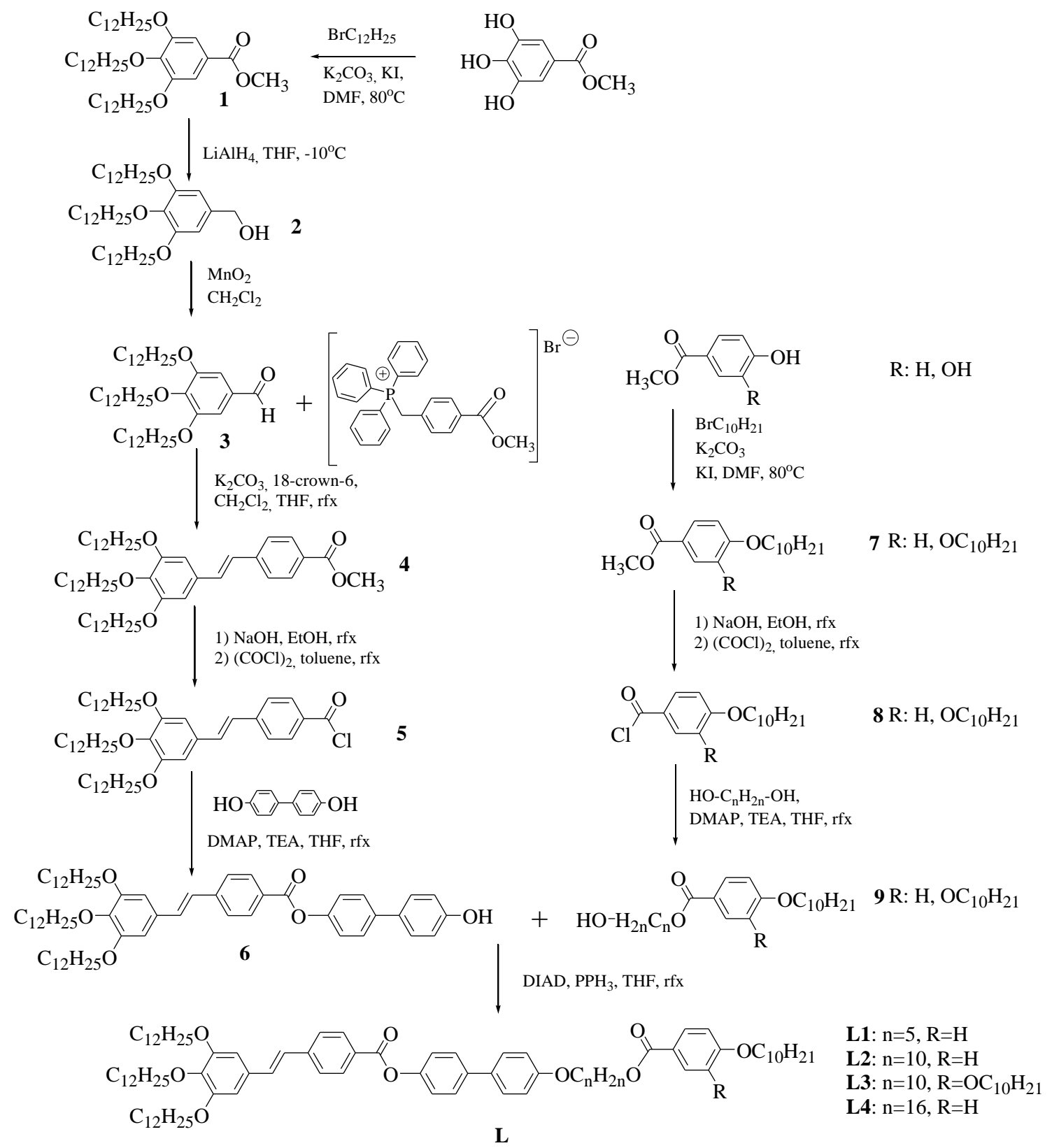

Scheme 1. Synthesis of the final compounds L 


\section{Synthesis of the 3,4,5-tridodecyloxybenzyl alcohol (2)}

Into the solution of $15 \mathrm{~g}(0,2 \mathrm{~mol})$ of methyl 3,4,5-tridodecyloxybenzoate (1) in $150 \mathrm{ml}$ of dry tetrahydrofuran cooled into $-10^{\circ} \mathrm{C}$ and kept under argon atmosphere a $20 \mathrm{ml}$ $(0,02 \mathrm{~mol})$ of $1 \mathrm{M}$ solution lithium aluminum hydride in tetrahydrofuran was adder dropwise. The mixture was stirred at room temperature for $24 \mathrm{~h}$. The reaction was terminated by adding methanol dropwise and the solution was filtered through celite. The solvent was evaporated and precipitate was dissolved in dichloromethane and washed with $5 \%$ hydrochloric acid in distilled water. Crude product was purified with chromatography column (eluent: toluen), yielded $12,9 \mathrm{~g}(98 \%)$ of white solid. Elemental analysis for $\mathrm{C}_{43} \mathrm{H}_{80} \mathrm{O}_{4}(\mathrm{M}=660,61) \mathrm{C} 78,12 ; \mathrm{H} 12,20$; \%, calc., found: $\mathrm{C} 78,16 ; \mathrm{H}$ 12,30\%; ${ }^{1} \mathrm{H}$ NMR $\left(200 \mathrm{MHz}, \mathrm{CDCl}_{3}\right) \delta: 6.65(2 \mathrm{H}, \mathrm{s}) ; 4.60(2 \mathrm{H}, \mathrm{d}, \mathrm{J}=5.9) ; 4.09-3.96$ $(6 \mathrm{H}, \mathrm{m}) ; 1.92-1.66(6 \mathrm{H}, \mathrm{m}) ; 1.58-1.14(54 \mathrm{H}, \mathrm{m}) ; 0.94-0.80(9 \mathrm{H}, \mathrm{m})$.

\section{Synthesis of 3,4,5-tridodecyloxybenzaldehyde (3)}

Into the solution of $15 \mathrm{~g}(0,02 \mathrm{~mol})$ of 3,4,5-tridodecyloxybenzyl alcohol (2) in dichloromethane 39,5g $(0,45 \mathrm{~mol})$ of activated manganese (IV) oxide was added. The reaction was stirred vigorously for $1 \mathrm{~h}$. The mixture was filtered through celite and the solvent was evaporated yielded 14,7g (99\%) of lightly yellow solid. Elemental analysis

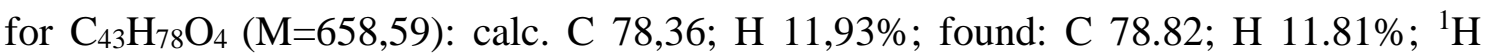
NMR: (200MHz $\left.\mathrm{CDCl}_{3}\right) \delta: 9.98(2 \mathrm{H}, \mathrm{s}) ; 6.73(2 \mathrm{H}, \mathrm{s}) ; 4.08-3.94(6 \mathrm{H}, \mathrm{m}) ; 1.92-1.66$ $(6 \mathrm{H}, \mathrm{m}) ; 1.58-1.14(54 \mathrm{H}, \mathrm{m}) ; 0.94-0.80(9 \mathrm{H}, \mathrm{m})$.

\section{Synthesis of the methyl 4-\{(E)-2-[3,4,5-tris(dodecyloxy)phenyl] ethenyl\}benzoate} (4):

Into the solution of $12 \mathrm{~g} \quad(24 \mathrm{mmol})$ of (4-methoxycarbonyl-benzyl)triphenylphosphonium bromide1 in $170 \mathrm{ml}$ of dichloromethane and $200 \mathrm{ml}$ of tetrahydrofuran $10,5 \mathrm{~g}(0.76 \mathrm{~mol})$ of anhydrous potassium carbonate and catalytic 
amount of 18-crown-6 was added in one portion. After 15 minutes to the vigorously stirred mixture a $15 \mathrm{~g}(22 \mathrm{mmol})$ of 3,4,5-trisdodecyloxybenzaldehyde (3), diluted in $30 \mathrm{ml}$ of dichloromethane, was added. The reaction mixture was refluxed for $48 \mathrm{~h}$ and next cooled down to the room temperature. An inorganic compounds was filtrated and solvents was removed. The crude product was recrystallized from ethanol and purified by chromatography column (eluent chloroform) to give $14,9 \mathrm{~g}(70 \%)$ of white solid. Elemental analysis for $\mathrm{C}_{52} \mathrm{H}_{86} \mathrm{O}_{5}(\mathrm{M}=790,65)$ : C 78.93; $\mathrm{H} 10.96 \%$; calc. found $\mathrm{C} 78.82$; H 11.01\%; ${ }^{1} \mathrm{H}$ NMR: $\left(200 \mathrm{MHz}, \mathrm{CDCl}_{3}\right) \delta: 8,01(2 \mathrm{H} ; \mathrm{d} ; \mathrm{J}=8,1) ; 7,53(2 \mathrm{H} ; \mathrm{d} ; \mathrm{J}=8,1)$; 7,12 (1H; d; J=16,1); 6,98 (1H; d; J=16,1); 6,73 (s; 2H); 4,08 - 3,94(6H; m); 3,91 (3H, s); $1,92-1,66(6 \mathrm{H} ; \mathrm{m}) 1,58-1,14(54 \mathrm{H} ; \mathrm{m}) ; 0,94-0,80(9 \mathrm{H} ; \mathrm{m}) ;{ }^{13} \mathrm{C} \mathrm{NMR}\left(50 \mathrm{MHz}, \mathrm{CDCl}_{3}\right) \delta$ : $167.10 ; 153.57 ; 142.15 ; 139.05 ; 132.12 ; 131.69 ; 130.22 ; 128.88 ; 126.72 ; 126.35 ; 105.69 ; 73.77$; $69.41 ; 52.26 ; 32.14 ; 30.56 ; 29.92 ; 29.88 ; 29.64 ; 29.59 ; 26.33 ; 22.91 ; 14.32 ;$

\section{Synthesis of 4-\{(E)-2-[3,4,5-tris(dodecyloxy)phenyl]ethenyl\}benzoyl chloride} (5):

Into the mixture of $15 \mathrm{~g}(19 \mathrm{mmol})$ of compound 4 in $250 \mathrm{ml}$ of ethanol a $37 \mathrm{~g}(0.92 \mathrm{~mol})$ of $\mathrm{KOH}$ diluted in $10 \mathrm{ml}$ of distilled water was added in portions. The reaction mixture was stirred under reflux for $12 \mathrm{~h}$. After cooling to the room temperature the precipitate was filtered and dried under vacuum for $24 \mathrm{~h}$ to give a $15,5 \mathrm{~g}(100 \%)$ of white solid. A $7 \mathrm{~g}(8,8 \mathrm{mmol})$ of dry product was dissolved in anhydrous toluene. Into the mixture $10 \mathrm{ml}$ $(0.11 \mathrm{~mol})$ of oxalyl chloride was added dropwise. The reaction mixture was stirred under reflux for $8 \mathrm{~h}$. The cooled mixture was filtrated to separate inorganic wastes and solution was concentrated to give bright yellow residue which was dried under vacuum and used in next reaction without any purification. Yield 7,1g (100\%). The product was used without purification. 


\section{Synthesis of 4'-hydroxybiphenyl-4-yl 4-\{(E)-2-[3,4,5tris(dodecyloxy)phenyl]}

ethenyl) benzoate (6):

Into the solution of $0,74 \mathrm{~g}(4 \mathrm{mmol})$ of the 4,4 'biphenol in $80 \mathrm{ml}$ of dry THF $2 \mathrm{ml}$ of dry pyridine and catalytic amount of DMAP was added. Next the solution of $1,2 \mathrm{~g}$ $(1,6 \mathrm{mmol})$ of chloride derivative (5) in $40 \mathrm{ml}$ of dry THF was added dropwise. The mixture was stirred at $70^{\circ} \mathrm{C}$ for $16 \mathrm{~h}$. The solvent was removed and the residue was purified by column chromatography (eluent: $\mathrm{CHCl}_{3}$ ) and recrystallized with ethanol to yield $0,9 \mathrm{~g}(60 \%)$ of white solid.Elemental analysis for $\mathrm{C}_{63} \mathrm{H}_{92} \mathrm{O}_{6}(\mathrm{M}=944,69)$ : calc. C 80.04; H 9,81\%; found C 80.26; H 9,95\%; ${ }^{1} \mathrm{H}$ NMR: (300MHz, $\left.\mathrm{CDCl}_{3}\right) \delta: 8,19(2 \mathrm{H} ; \mathrm{d} ; \mathrm{J}=8,7) ; 7,61$ $(2 \mathrm{H} ; \mathrm{d} ; \mathrm{J}=8,7) ; 7,54(2 \mathrm{H} ; \mathrm{d} ; \mathrm{J}=8,7 \mathrm{~Hz}) ; 7,41(2 \mathrm{H} ; \mathrm{d} ; \mathrm{J}=8,7) ; 7,24(2 \mathrm{H} ; \mathrm{d} ; \mathrm{J}=8,7) ; 7,17(1 \mathrm{H} ; \mathrm{d}$; $\mathrm{J}=16,2) ; 7,02(1 \mathrm{H} ; \mathrm{d} ; \mathrm{J}=16,2) ; 6,84(2 \mathrm{H} ; \mathrm{d} ; \mathrm{J}=8,7) ; 6,76(2 \mathrm{H} ; \mathrm{s}) ; 5,46($ br. $1 \mathrm{H} ; \mathrm{s}) ; 4,08-3,96$ $(6 \mathrm{H} ; \mathrm{m}) ; 1,89-1,71(6 \mathrm{H} ; \mathrm{m}) 1,57-1,43(6 \mathrm{H} ; \mathrm{m}) ; 1,43-1,20(48 \mathrm{H}) ; 0,93-0,83(9 \mathrm{H} ; \mathrm{m}) ;{ }^{13} \mathrm{C}$ NMR $\left(75 \mathrm{MHz}, \mathrm{CDCl}_{3}\right) \delta 165.38 ; 155.44 ; 153.38 ; 149.87 ; 142.76 ; 138.86 ; 138.72 ; 132.82 ;$ $132.00 ; 131.89 ; 130.71 ; 128.30 ; 127.88 ; 127.73 ; 126.41 ; 126.34 ; 121.92 ; 115.72 ; 105.56 ; 73.62$; $69.25 ; 31.94 ; 30.33 ; 29.77 ; 29.72 ; 29.68 ; 29.62 ; 29.44 ; 29.41 ; 29.39 ; 26.14 ; 22.70 ; 14.12$;

\section{Synthesis of methyl 4-decyloxybenzoate (7)}

The procedure of obtaining of compound $\mathbf{7}$ was the same as for compound 1. Elemental analysis for $\mathrm{C}_{18} \mathrm{H}_{28} \mathrm{O}_{3}(\mathrm{M}=292.2)$ : calc. $\mathrm{C} 73.93, \mathrm{H} \mathrm{9.65 \%}$; found $\mathrm{C} 73.64, \mathrm{H} \mathrm{9.45 \% ;}{ }^{1} \mathrm{H}$ $\operatorname{NMR}\left(200 \mathrm{MHz}, \mathrm{CDCl}_{3}\right) \delta: 7.97(2 \mathrm{H}, \mathrm{d}, \mathrm{J}=9.0), 6.89(2 \mathrm{H}, \mathrm{d}, \mathrm{J}=9.0), 3.98(2 \mathrm{H}, \mathrm{t}, \mathrm{J}=$ 6.3), $3.87(3 \mathrm{H}, \mathrm{s}), 1.91-1.68(2 \mathrm{H}, \mathrm{m}), 1.54-1.16(14 \mathrm{H}, \mathrm{m}), 0.96-0.78(3 \mathrm{H}, \mathrm{m})$

\section{Synthesis of 4-decyloxybenzoyl chloride (8)}

Compound $\mathbf{8}$ was obtained in the same synthesis procedure, conditions and molar ratio as used for compound $\mathbf{5}$. The product was used without purification. 


\section{Synthesis of 5-hydroxypentyl 4-(decyloxy)benzoate (9)}

Into the solution of $1.3 \mathrm{~g}(12.6 \mathrm{mmol})$ of the 1,5 -pentanediol in $100 \mathrm{ml}$ of dry THF $2 \mathrm{ml}$ of dry $\mathrm{NEt}_{3}$ and catalytic amount of DMAP was added. Next the solution of $1.5 \mathrm{~g}$ (5.05mmol) of compound $7 \mathrm{in} 50 \mathrm{ml}$ of dry THF was added dropwise. The mixture was stirred at $70^{\circ} \mathrm{C}$ for $16 \mathrm{~h}$. The solvent was removed and the residue was purified by column chromatography (eluent: $\mathrm{CHCl}_{3}$ ) and recrystallized with ethanol to yield $1,0 \mathrm{~g}$ (55\%) of white solid. Elemental analysis for $\mathrm{C}_{22} \mathrm{H}_{36} \mathrm{O}_{4},(\mathrm{M}=364,26)$ : calc. C 72.49; $\mathrm{H}$ 9.95\%; found C 72.41; $\mathrm{H} 9.99 \% ;{ }^{1} \mathrm{H}$ NMR $\left(200 \mathrm{MHz}, \mathrm{CDCl}_{3}\right) \delta: 7.88(2 \mathrm{H} ; \mathrm{d} ; \mathrm{J}=9.0) ; 6.90(2 \mathrm{H} ; \mathrm{d} \mathrm{J}=9.0)$; $4.29(2 \mathrm{H} ; \mathrm{t} ; \mathrm{J}=6.3) ; 4.00(2 \mathrm{H} ; \mathrm{t} ; \mathrm{J}=6.3) ; 3.68(2 \mathrm{H} ; \mathrm{t} ; \mathrm{J}=6.0) ; 1.90-1.69(2 \mathrm{H} ; \mathrm{m}) ; 1.70-1.40$ $(4 \mathrm{H} ; \mathrm{m}) ; 1.41-1.02(16 \mathrm{H} ; \mathrm{m}) ; 0.95-0.76(3 \mathrm{H} ; \mathrm{m})$;

\section{Synthesis of 5-(\{4'-[(4-\{(E)-2-[3,4,5-tris(dodecyloxy)phenyl]ethenyl\}benzoyl) oxy] biphenyl-4-yl\}oxy)pentyl 4-(decyloxy)benzoate (L1)}

Into the $100 \mathrm{mg}(0,10 \mathrm{mmol})$ of compound $\mathbf{6}, 50 \mathrm{~g}(0.13 \mathrm{mmol})$ compound 9 and $36 \mathrm{mg}$ $(0.13 \mathrm{mmol})$ of triphenylphosphine dissolved in $15 \mathrm{ml}$ of dry THF cooled to the $0^{\circ} \mathrm{C} 32 \mu \mathrm{l}$ $(0.13 \mathrm{mmol})$ of DIAD wad added dropwise. The reaction mixture was stirred at $70^{\circ} \mathrm{C}$ for 12h. The solvent was removed and the crude product was purified by chromatography column (eluent: toluene - hexane, 1:1), yield 90mg (66\%). Elemental analysis for $\mathrm{C}_{85} \mathrm{H}_{126} \mathrm{O}_{9},(\mathrm{M}=1290,94)$ : calc. $\mathrm{C} 79.02 ; \mathrm{H} 9.83 \%$; found: $\mathrm{C} 79.14 ; \mathrm{H} 9.89 \% ;{ }^{1} \mathrm{H}$ NMR: $\left(300 \mathrm{MHz}, \mathrm{CDCl}_{3}\right) \delta: 8.19(2 \mathrm{H} ; \mathrm{d} ; \mathrm{J}=8.4) ; 7.98(2 \mathrm{H} ; \mathrm{d} ; \mathrm{J}=9.0) ; 7.67-7.55(4 \mathrm{H} ; \mathrm{m}) ; 7.51(2 \mathrm{H} ; \mathrm{d}$ $\mathrm{J}=8.7) ; 7.26(2 \mathrm{H} ; \mathrm{d} ; \mathrm{J}=8.7) ; 7.17(1 \mathrm{H} ; \mathrm{d} ; \mathrm{J}=16.2) ; 7.02(1 \mathrm{H} ; \mathrm{d} ; \mathrm{J}=16.2) ; 6.97(2 \mathrm{H} ; \mathrm{d} ; \mathrm{J}=8.7)$; $6.84(2 \mathrm{H} ; \mathrm{d} ; \mathrm{J}=8.7) ; 6.76(2 \mathrm{H} ; \mathrm{s}) ; 4,33(2 \mathrm{H} ; \mathrm{t} ; \mathrm{J}=6.3) ; 4.09-3.94(10 \mathrm{H} ; \mathrm{m}) ; 1.96-1,61(12 \mathrm{H}$; $\mathrm{m}) ; 1.54-1.42(10 \mathrm{H} ; \mathrm{m}) ; 1.42-1.17(60 \mathrm{H} ; \mathrm{m}) ; 0.95-0.82(12 \mathrm{H} ; \mathrm{m}) ;{ }^{13} \mathrm{C}$ NMR $(75 \mathrm{MHz}$ $\left.\mathrm{CDC}_{13}\right) \delta: 166.47 ; 165.08 ; 162.92 ; 153.37 ; 142.66 ; 138.94 ; 138.68 ; 132.87 ; 131.92 ; 131.80 ;$ $131.52 ; 130.66 ; 128.12 ; 127.97 ; 127.73 ; 126.38 ; 126.30 ; 122.54 ; 121.90 ; 114.81 ; 114.03 ;$ $105.54 ; 73.59 ; 69.37 ; 69.22 ; 68.20 ; 67.75 ; 67.68 ; 64.49 ; 31.92 ; 31.88 ; 30.34 ; 29.76 ; 29.70$; 
$29.66 ; 29.53 ; 29.42 ; 29.39 ; 29.37 ; 29.30 ; 29.10 ; 28.94 ; 28.57 ; 26.12 ; 25.97 ; 22.73 ; 22.69$; 14.11;

L2 Elemental analysis for $\mathrm{C}_{90} \mathrm{H}_{136} \mathrm{O}_{9}(\mathrm{M}=1361,02)$ : calc. C 79.36; $\mathrm{H} 10.06 \%$; found $\mathrm{C}$ 79.32; H 10.13\%; ${ }^{1} \mathrm{H}$ NMR: $\left(200 \mathrm{MHz}, \mathrm{CDCl}_{3}\right) \delta: 8.19(2 \mathrm{H} ; \mathrm{d} ; \mathrm{J}=8.4) ; 7.98(2 \mathrm{H} ; \mathrm{d}$; $\mathrm{J}=8.8) ; 7.67-7.55(4 \mathrm{H} ; \mathrm{m}) ; 7.51(2 \mathrm{H} ; \mathrm{d} ; \mathrm{J}=8.8) ; 7.26(2 \mathrm{H} ; \mathrm{d} ; \mathrm{J}=8.4) ; 7.18(1 \mathrm{H} ; \mathrm{d}$; $\mathrm{J}=15.9) ; 7.10-6.93(3 \mathrm{H} ; \mathrm{m}) ; 6.90(2 \mathrm{H} ; \mathrm{d} ; \mathrm{J}=8.8) ; 6.76(2 \mathrm{H} ; \mathrm{s}) ; 4,28(2 \mathrm{H} ; \mathrm{t} ; \mathrm{J}=6.6)$; $4.10-3.92(10 \mathrm{H} ; \mathrm{m}) ; 1.92-1.66(12 \mathrm{H} ; \mathrm{m}) ; 1.57-1.18(84 \mathrm{H} ; \mathrm{m}) ; 0.96-0.81(12 \mathrm{H}$; $\mathrm{m}) ;{ }^{13} \mathrm{C}$ NMR $\left(50 \mathrm{MHz}, \mathrm{CDC}_{13}\right) \delta: 166.70 ; 165.26 ; 163.06 ; 158.96 ; 153.57 ; 150.08 ;$ $142.84 ; 139.12 ; 138.99 ; 132.92 ; 132.10 ; 132.00 ; 131.69 ; 130.86 ; 128.30 ; 128.20 ; 127.91$; $126.50 ; 122.10 ; 115.00 ; 114.20 ; 105.71 ; 73.76 ; 69.41 ; 68.38 ; 64.97 ; 32.13 ; 30.54 ; 29.87 ; 29.74$ $29.63 ; 29.30 ; 28.97 ; 26.32 ; 26.24 ; 22.89 ; 14.31$;

L3 Elemental analysis for $\mathrm{C}_{100} \mathrm{H}_{156} \mathrm{O}_{10}(\mathrm{M}=1517,17)$ : calc. C 79.11; $\mathrm{H} 10.36 \%$; found $\mathrm{C}$ 79.19; H 10.42\%; ${ }^{1} \mathrm{H}$ NMR: $\left(300 \mathrm{MHz}, \mathrm{CDCl}_{3}\right) \delta: 8.19(2 \mathrm{H} ; \mathrm{d} ; \mathrm{J}=8.4) ; 7.67$-7.56 (5H; m); $7.54(1 \mathrm{H} ; \mathrm{d} ; \mathrm{J}=1.8) ; 7.51(2 \mathrm{H} ; \mathrm{d} ; \mathrm{J}=8.7) ; 7.26(2 \mathrm{H} ; \mathrm{d} ; \mathrm{J}=8.7) ; 7.17(1 \mathrm{H} ; \mathrm{d} ; \mathrm{J}=16.2)$; $7.02(1 \mathrm{H} ; \mathrm{d} ; \mathrm{J}=16.2) ; 6.97(2 \mathrm{H} ; \mathrm{d} ; \mathrm{J}=9.0) ; 6.86(1 \mathrm{H} ; \mathrm{d} ; \mathrm{J}=8.4) ; 6.76(2 \mathrm{H} ; \mathrm{s}) ; 4,28(2 \mathrm{H} ; \mathrm{t}$; $\mathrm{J}=6.6) ; 4.09-3.94(12 \mathrm{H} ; \mathrm{m}) ; 1.90-1.69(12 \mathrm{H} ; \mathrm{m}) ; 1.55-1.42(14 \mathrm{H} ; \mathrm{m}) ; 1.41-1.18$ $(80 \mathrm{H} ; \mathrm{m}) ; 0.95-0.82(15 \mathrm{H} ; \mathrm{m}) ;{ }^{13} \mathrm{C} \mathrm{NMR}\left(75 \mathrm{MHz}, \mathrm{CDC}_{13}\right) \delta: 166.62 ; 165.08 ; 158.79$; $153.40 ; 153.14 ; 149.91 ; 148.50 ; 142.67 ; 138.97 ; 138.71 ; 132.76 ; 131.94 ; 131.82$ $130.68 ; 128.12 ; 128.00 ; 127.73 ; 126.32 ; 123.44 ; 122.80 ; 121.92 ; 114.82 ; 114.35 ;$ $111.92 ; 105.57 ; 73.59 ; 69.30 ; 69.24 ; 69.02 ; 68.10 ; 64.90 ; 31.94 ; 30.37 ; 29.78 ; 29.73 ;$ $29.68 ; 29.64 ; 29.61 ; 29.45 ; 29.39 ; 29.29 ; 29.20 ; 29.09 ; 28.80 ; 26.14 ; 26.06 ; 26.03$; $25.99 ; 22.71 ; 14.13$

L4 Elemental analysis for $\mathrm{C}_{96} \mathrm{H}_{148} \mathrm{O}_{9},(\mathrm{M}=1445,11)$ : calc. C 79.73; $\mathrm{H} 10.31 \%$; found $\mathrm{C}$ 79.69; H 10.44\%; ${ }^{1} \mathrm{H}$ NMR: (300MHz, $\left.\mathrm{CDCl}_{3}\right) \delta: 8.19(2 \mathrm{H} ; \mathrm{d} ; \mathrm{J}=8.4) ; 7.98(2 \mathrm{H} ; \mathrm{d}$; 
$\mathrm{J}=9.0) ; 7.66-7.56(4 \mathrm{H} ; \mathrm{m}) ; 7.51(2 \mathrm{H} ; \mathrm{d} ; \mathrm{J}=8.7) ; 7.26(2 \mathrm{H} ; \mathrm{d} ; \mathrm{J}=8.7) ; 7.17(1 \mathrm{H} ; \mathrm{d} ;$ $\mathrm{J}=16.2) ; 7.02(1 \mathrm{H} ; \mathrm{d} ; \mathrm{J}=16.2) ; 6.97(2 \mathrm{H} ; \mathrm{d} ; \mathrm{J}=8.7) ; 6.70(2 \mathrm{H} ; \mathrm{d} ; \mathrm{J}=9.0) ; 6.76(2 \mathrm{H} ; \mathrm{s})$; $4.27(2 \mathrm{H} ; \mathrm{t} ; \mathrm{J}=6.6) ; 4.08-3.95(10 \mathrm{H} ; \mathrm{m}) ; 1.89-1.68(12 \mathrm{H} ; \mathrm{m}) ; 1.54-1.42(12 \mathrm{H} ; \mathrm{m})$ $1.41-1.18(80 \mathrm{H} ; \mathrm{m}) ; 0.94-0.83(12 \mathrm{H} ; \mathrm{m}) ;{ }^{13} \mathrm{C} \mathrm{NMR}\left(75 \mathrm{MHz}, \mathrm{CDC}_{13}\right) \delta: 166.57$ $165.09 ; 162.89 ; 158.87 ; 153.40 ; 139.00 ; 131.95 ; 131.52 ; 130.69 ; 128.13 ; 127.75$ $126.32 ; 122.71 ; 121.92 ; 114.84 ; 114.03 ; 105.58 ; 73.60 ; 69.26 ; 68.21 ; 68.14 ; 64.82 ;$ $31.96 ; 31.91 ; 30.38 ; 29.79 ; 29.73 ; 29.69 ; 29.63 ; 29.62 ; 29.57 ; 29.46 ; 29.42 ; 29.40$; $29.33 ; 29.13 ; 28.81 ; 26.15 ; 26.09 ; 26.00 ; 22.72 ; 14.14$;

\section{Additional experimental results}

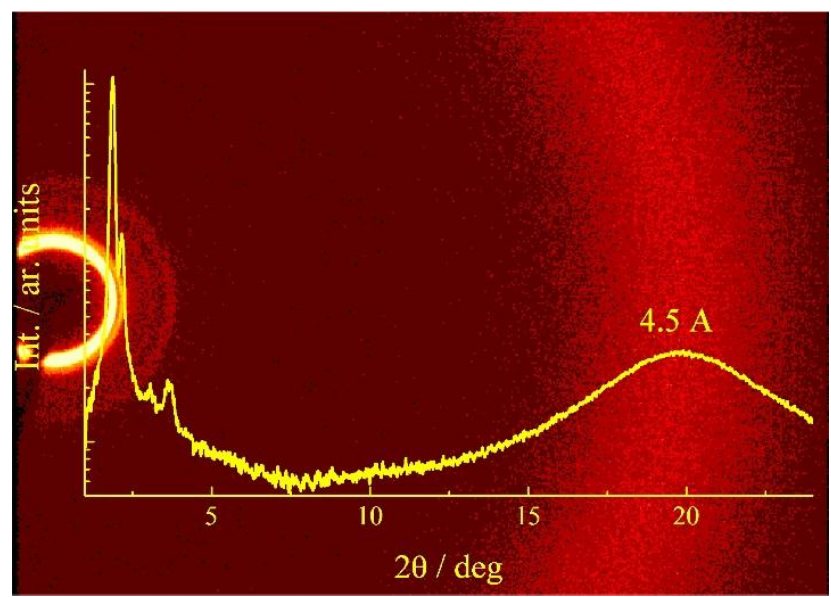

Figure S1. Broad angle xrd pattern for cubic phase of compound L1. Diffused signal at wide angle range, corresponding to periodicity $4.5 \mathrm{~A}$ reflects short-range positional correlations between neighbouring molecules. 


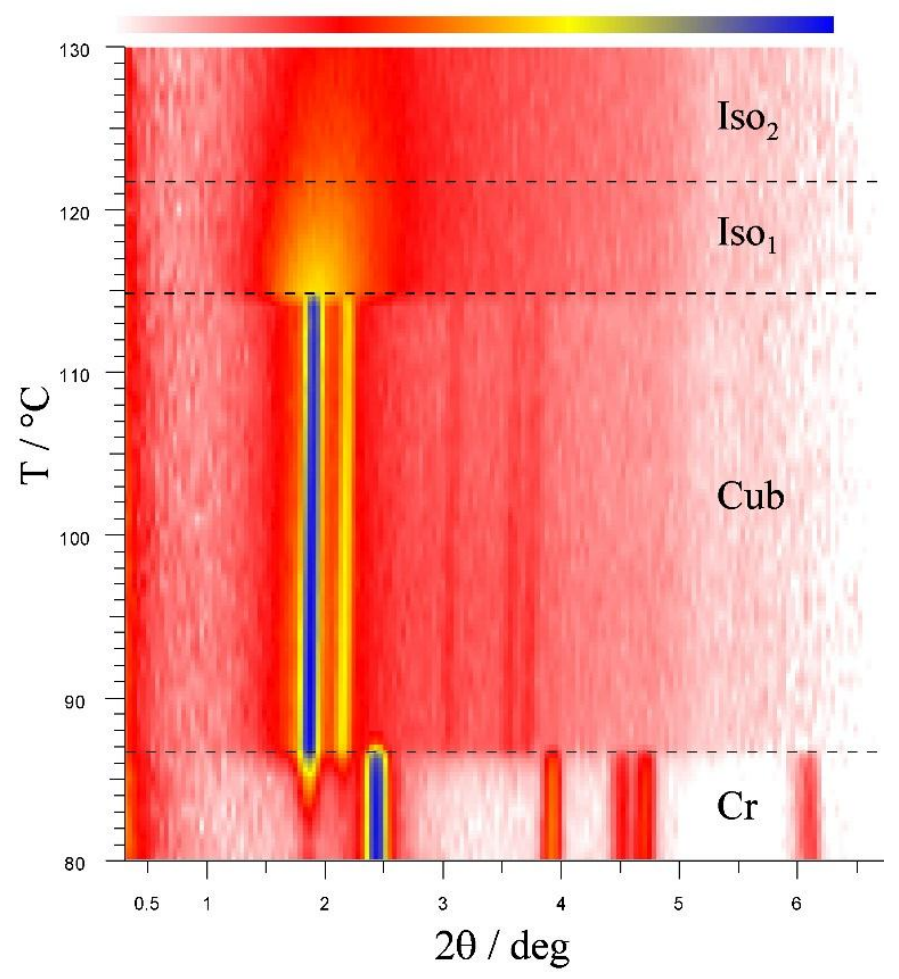

Figure S2. Temperature evolution of small angle xrd pattern for compound L1, measured on heating. Signal intensities are coded by colour. Note the temperature range (above cubic phase) of isotropic liquid with very pronounced fluctuations manifesting as intense broad signal.

Table S1. Positions of diffraction signals $\left(\mathrm{d}_{\mathrm{obs}}\right)$ observed in Cub phase of compound $\mathbf{L 1}$ at $95{ }^{\circ} \mathrm{C}$. Calculated values and indexes were obtained assuming crystallographic lattice with Ia3d symmetry and unit cell parameter a $=115.6 \mathrm{~A}$.

\begin{tabular}{l|l|l}
$\mathbf{d}_{\text {obs }}$ & $\mathbf{d}_{\text {calc }}$ & $(\mathbf{h k l})$ \\
\hline $\mathbf{4 7 . 2 0}$ & 47.19 & $(211)$ \\
\hline $\mathbf{4 0 . 8 8}$ & 40.87 & $(022)$ \\
\hline $\mathbf{2 8 . 9 0}$ & 28.90 & $(004)$ \\
\hline $\mathbf{2 4 . 6 7}$ & 24.65 & $(332)$ \\
\hline 23.58 & 23.60 & $(422)$ \\
\hline
\end{tabular}

Table S2. Positions of diffraction signals (dobs) observed in Cub phase of compound L2 at $65{ }^{\circ} \mathrm{C}$. Calculated values and indexes were obtained assuming crystallographic lattice with Ia3d symmetry and unit cell parameter $\mathrm{a}=119.1 \mathrm{~A}$.

\begin{tabular}{l|l|l}
$\mathbf{d}_{\text {obs }}$ & $\mathbf{d}_{\text {calc }}$ & $(\mathbf{h k l})$ \\
\hline $\mathbf{4 8 . 6 2}$ & 48.63 & $(211)$ \\
\hline $\mathbf{4 2 . 1 3}$ & 42.11 & $(022)$ \\
\hline $\mathbf{2 9 . 7 5}$ & 29.78 & $(004)$ \\
\hline $\mathbf{2 6 . 7 0}$ & 26.63 & $(042)$ \\
\hline $\mathbf{2 5 . 4 0}$ & 25.40 & $(332)$ \\
\hline $\mathbf{2 4 . 3 3}$ & 24.31 & $(422)$ \\
\hline
\end{tabular}


Table S3. Positions of diffraction signals $\left(\mathrm{d}_{\mathrm{obs}}\right)$ observed in Colh phase of compound $\mathbf{L 3}$ at $75{ }^{\circ} \mathrm{C}$. Calculated values and indexes were obtained assuming $2 \mathrm{D}$ hexagonal lattice with unit cell parameter $\mathrm{a}=52.10 \mathrm{~A}$.

\begin{tabular}{|l|l|l}
$\mathbf{d}_{\text {obs }}$ & $\mathbf{d}_{\text {calc }}$ & $($ hk $)$ \\
\hline $\mathbf{4 5 . 1 2}$ & 45.12 & $(10)$ \\
\hline $\mathbf{2 8 . 7 6}$ & & \\
\hline $\mathbf{2 6 . 0 6}$ & 26.05 & $(11)$ \\
\hline $\mathbf{2 2 . 6 1}$ & 22.56 & $(20)$ \\
\hline
\end{tabular}

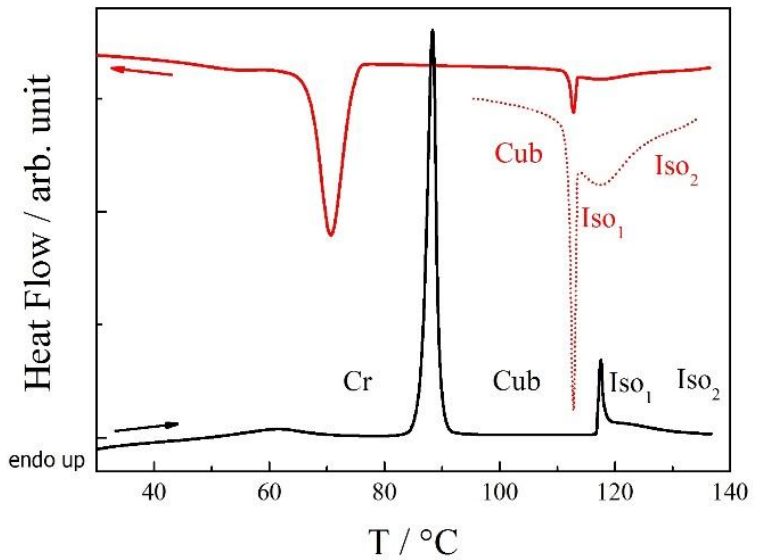

Figure S3. DSC thermograms recorded on heating and cooling runs for compound L1. Transition between two isotropic liquids is clearly visible.

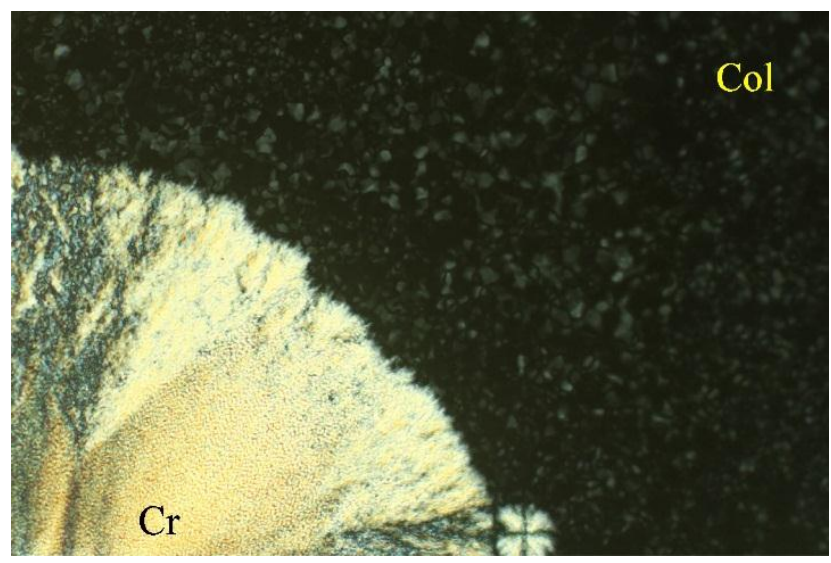

Figure S4. Optical texture obtained on cooling a sample of compound L3, showing coexistence of solid crystal and unidentified columnar phase. 


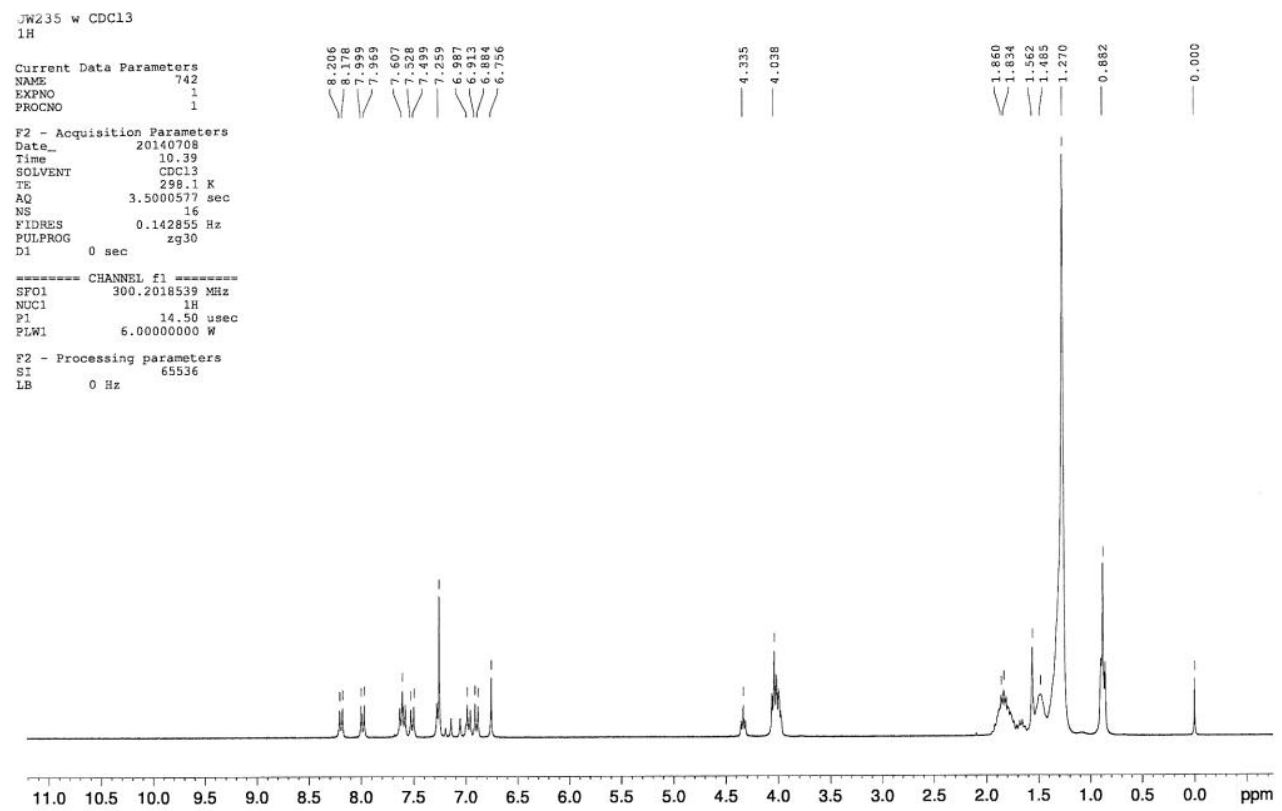

Figure S5. ${ }^{1} \mathrm{H}$ NMR spectrum for compound $\mathbf{L 1}$

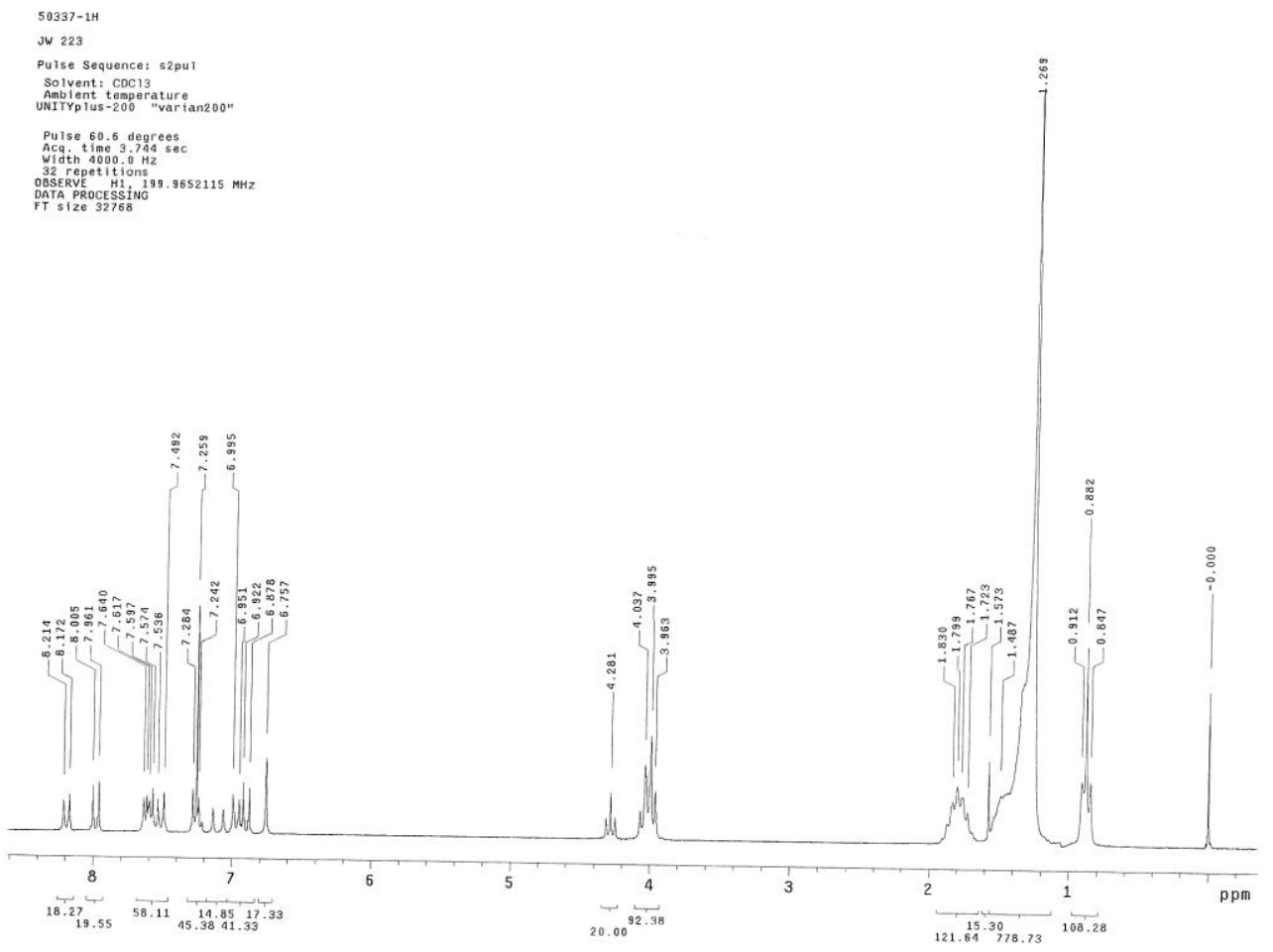

Figure S6. ${ }^{1} \mathrm{H}$ NMR spectrum for compound $\mathbf{L 2}$ 

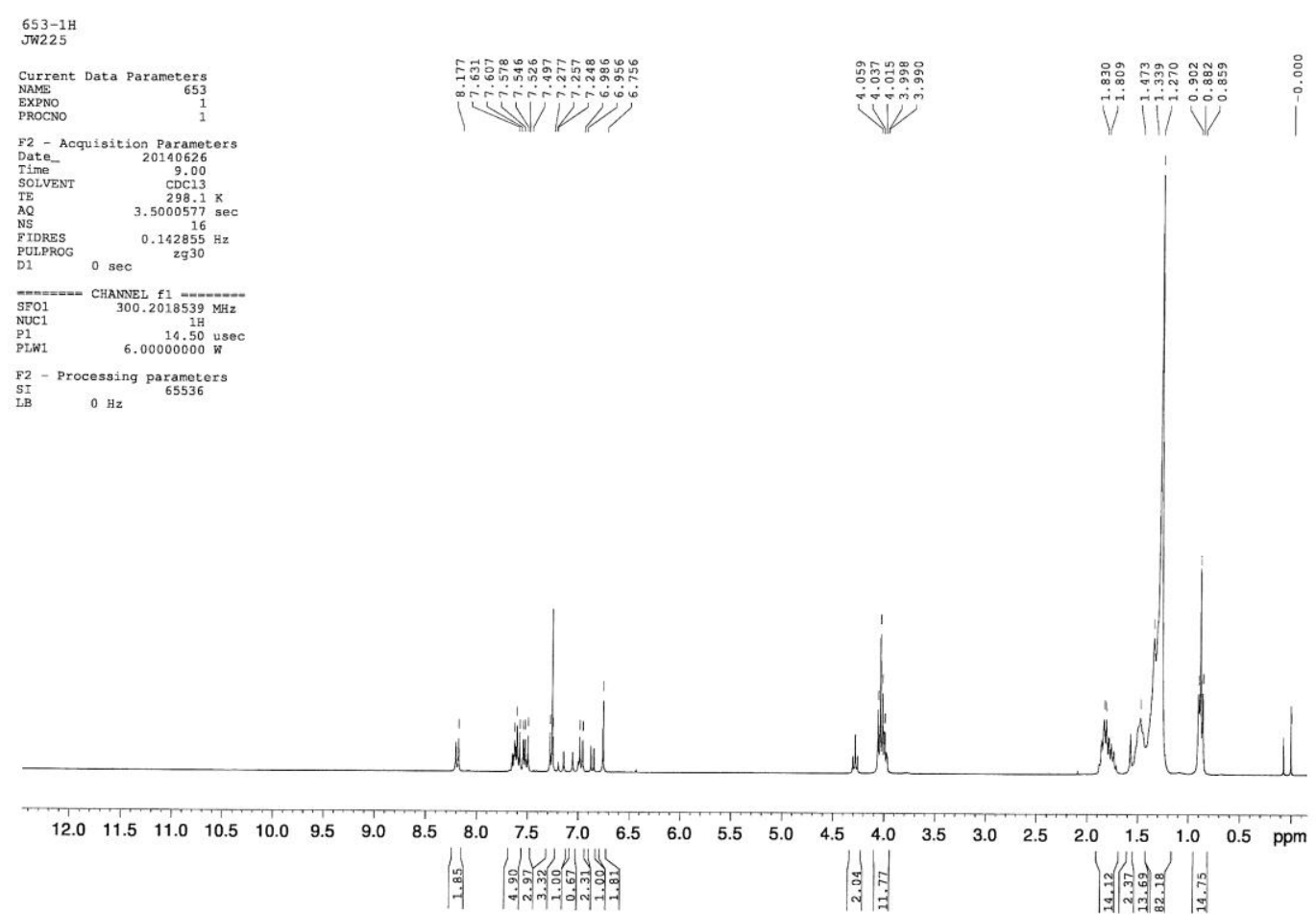

Figure S7. ${ }^{1} \mathrm{H}$ NMR spectrum for compound $\mathbf{L 3}$

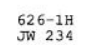

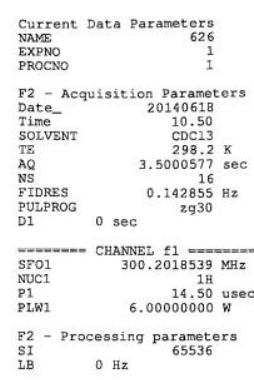

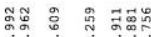

Vi i

iV

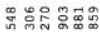

$\stackrel{\circ}{\circ}$
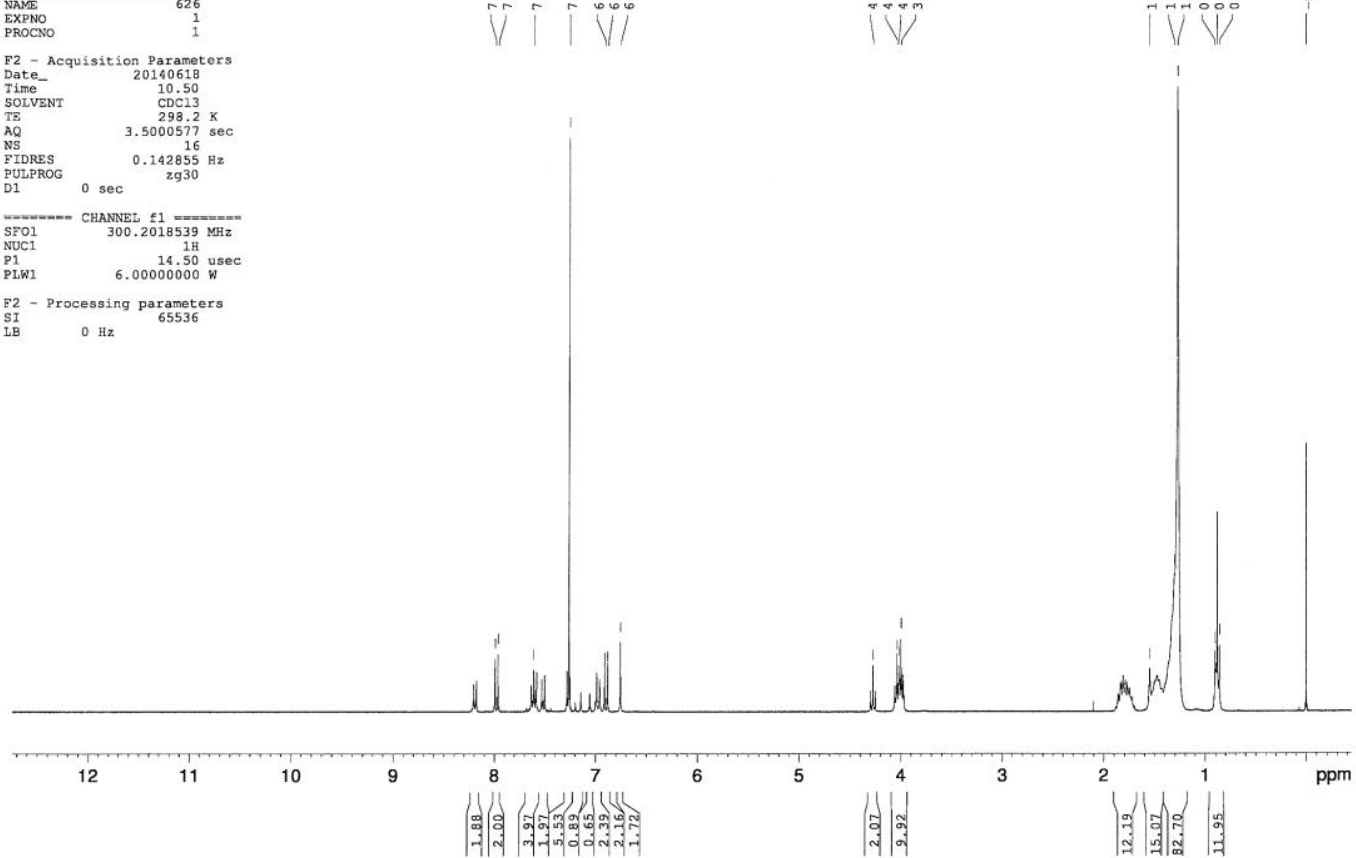

Figure S8. ${ }^{1}$ H NMR spectrum for compound $\mathbf{L} 4$ 


\section{References:}

1. J. M. Wolska, D. Pociecha, J. Mieczkowski and E. Gorecka, Soft Matter, 2013, 9, 3005 